\title{
Sub-Poissonian photocurrent statistics: Theory and undergraduate experiment
}

\author{
A. C. Funk* and M. Beck** \\ Dept. of Physics \\ Reed College \\ Portland, OR 97202
}

\begin{abstract}
We present the results of an experiment in which we observe a subPoissonian photocurrent. This photocurrent is generated by detecting the light emitted from a high-quantum-efficiency light-emitting diode driven by a high-impediance current source. We also present classical, semi-classical and quantum mechanical theories of photoelectric detection. The observed sub-Poissonian statistics are in agreement with quantum mechanical predictions, but are unexplainable using the classical and semi-classical theories. The experiment uses only equipment already found in most undergraduate laboratories, making this a nice experiment to demonstrate a purely quantum mechanical effect.
\end{abstract}

*Present Address: Dept. of Physics, Univ. of Oregon, Eugene, OR 97403

**Present Address: Dept. of Physics, Whitman College, Walla Walla, WA 99362;beckmk@whitman.edu 


\section{Introduction}

The statistical properties of light beams have been of interest to physicists since at least the end of the last century. This interest arises naturally from attempts to answer the question; "Is light a particle or a wave?" The arguments in favor of either interpretation are numerous, and many scientists have hoped that a deeper understanding of the full statistics of different types of light beams could help illuminate the answer to this seemingly unanswerable question.

It was Einstein who first applied Plank's radiation formula to the problem of the energy fluctuations of blackbody radiation in an enclosure at thermal equilibrium. [1, 2] We will call $E_{\nu}$ the energy of the radiation at frequency $\nu$ in a small frequency interval $d \nu$. By using Plank's blackbody radiation law, Einstein showed that the variance of the energy fluctuations is given by [3]

$$
\left\langle\left(\Delta E_{\nu}\right)^{2}\right\rangle=h \nu\left\langle E_{\nu}\right\rangle+\frac{c^{3}}{8 \pi \nu^{2} d \nu V}\left\langle E_{\nu}\right\rangle^{2}
$$

where $V$ is the volume of the enclosure.

As Einstein recognized, the first term corresponds to the fluctuations of classical particles of energy $h \nu$, while the second term corresponds to the fluctuations of classical waves. This second term alone is obtained if the RayleighJeans formula for blackbody radiation is used. Thus, as far back as 1909 it was realized that light fluctuations have two distinct components: a wave-like component, and a particle-like component. 
Here we will not directly discuss the question of the wave-particle duality of light. The main question we will attempt to address is, "When is it necessary to treat light fields as quantum mechanical objects?" The fully quantum mechanical model of light detection is the only model in agreement with all experiments to date. However, in most cases it is not necessary to treat the field quantum mechanically, and classical or semi-classical detection theory is sufficient to describe experimental results. Here we will describe the basic elements of classical, semi-classical and quantum mechanical photodetection theory. We will describe how they are similar, but also how they are different. Particular attention will be paid to the circumstances when the quantum model allows for predictions that cannot occur in the other two models.

We then present the results of an experiment that is in agreement with quantum mechanical detection theory, but violates classical and semi-classical theories. In this experiment, we observe the statistical properties of the light emitted by a high-quantum-efficiency light-emitting diode (LED). We show that under certain conditions, the fluctuations of the detected light are smaller than those allowed by classical and semi-classical photodetection theory. This noise reduction indicates that the light field generated by the LED is purely quantum mechanical, and has no classical analog.

The results of this experiment are not new, having been previously reported. [4, 5, 6] However, we have simplified the apparatus necessary for observing a convincing quantum mechanical effect $(0.7 \mathrm{~dB})$. The necessary 
equipment will be found in most undergraduate laboratories. Recently an undergraduate experiment for observing Poissoninan and Bose-Einstein photon statistics has been reported. [7] The experiment we describe here provides a nice complement to this previous work.

As will be seen, probably the most important aspect to this experiment is the calibration of the detectors, the specifics of which are not often reported in the literature. In keeping with the pedagogical aspect of this paper, we will carefully outline our calibration procedure.

\section{Theory}

With the development of quantum mechanics, it has become apparent that it is important to discuss the statistics of measured quantities. For light beams, the light itself is not measured, but rather one measures the photocurrent from a detector on which the light beam impinges. Thus, a theory of light fluctuations describes the statistics of the photocurrent, and these photocurrent statistics are what must be compared to experiments. In order to treat the detection of light, one must have a model for both the light and the detector. Models fall into three general categories: classical (both the light and the detector are treated classically), semi-classical (light is a classical wave, but the detector is treated quantum mechanically), and quantum mechanical (both the light field and the detector are treated quantum mechanically). 


\subsection{Classical and Semi-Classical Theories}

We begin with a classical model developed by Mandel in the late 1950's; [8, 9] this model is chosen for two reasons: the first is that it successfully described all experimental results up until the 1970's. The second is that this model yields results identical with the semi-classical model, giving it a special place among purely classical models. This second feature also allows us to state the semi-classical results without having to go into great detail.

Consider an optical detector that accepts a classical light wave as its input, and emits electrons as its output. The details of the interaction between the light and the electrons are unimportant, but we make three assumptions about the nature of the output photoelectrons. The first is that the emission of each electron is a random event, and the probability $P(1, t, t+d t)$ of one electron being emitted in a short time interval from $t$ to $t+d t$ is

$$
P(1, t, t+d t)=\gamma I(t) d t .
$$

In Eq. (2), $I(t)$ is the classical intensity of the incoming light beam and $\gamma$ is the efficiency of the detector (having units of 1 /energy). The second assumption is that the time interval $d t$ can be chosen small enough to ensure that at most one electron emission occurs (i.e., only 0 or 1 electrons are emitted within $d t$ ). The last assumption is that the number of photoemissions in nonoverlaping time intervals are statistically independent. Given these assumptions, it is possible to show that the probability $P(n, t, t+d t)$ of $n$ photoevents occurring 
in a macroscopic time interval of width $d t$ is given by $[8,9]$

$$
P(n, t, t+d t)=\frac{(\gamma W)^{n}}{n !} e^{-\gamma W}
$$

where $W$ is the integrated intensity

$$
W=\int_{t}^{t+d t} I\left(t^{\prime}\right) d t^{\prime}
$$

In general, the light intensity $I(t)$ is a random process, and no more can be said about the photoelectron statistics determined by Eq. (3) without specifying the form of the intensity fluctuations. In other words, $W$ is a random variable, and Eq. (3) must be considered to be a conditional probability distribution of the form $P(n \mid W)$ - the number of counts $n$ is conditioned on knowledge of $W$. Since $W$ is not explicitly known, what is actually measured in experiments is the unconditional probability $[8,9]$

$$
\begin{aligned}
P(n) & =\int_{0}^{\infty} P(n \mid W) p_{W}(W) d W \\
& =\int_{0}^{\infty} \frac{(\gamma W)^{n}}{n !} e^{-\gamma W} p_{W}(W) d W
\end{aligned}
$$

where $p_{W}(W)$ is the probability density of the integrated intensity $W$. Here we have eliminated the explicit dependence on the time window from our notation. It will be understood that $P(n)$ represents the probability of obtaining $n$ of photocounts in a particular time interval.

In our experiments, we do not measure the full probability distribution $P(n)$, but rather moments of this distribution. It can be shown using Eq. (5) that the mean and variance of the number of photocounts are given by

$$
\langle n\rangle=\gamma\langle W\rangle
$$


and

$$
\begin{aligned}
\left\langle(\Delta n)^{2}\right\rangle & =\gamma\langle W\rangle+\gamma^{2}\left\langle(\Delta W)^{2}\right\rangle \\
& =\langle n\rangle+\gamma^{2}\left\langle(\Delta W)^{2}\right\rangle .
\end{aligned}
$$

Equation (7) shows that there are two contributions to the variance of the number of photocounts. There is a term linear in the integrated intensity $W$ (i.e., the energy incident on the detector in the time interval $d t$ ), and a term quadratic in $W$. This should be reminiscent of Einstein's expression for the energy fluctuations of a blackbody [Eq. (1)]. The first term represents noise introduced by the random emission of photoelectrons, which is analogous to Einstein's particle noise. We refer to this term as the "shot-noise". The second term comes from the fluctuations of the wave incident on the detector, and is the classically expected result that would be obtained in the absence of any particle-like fluctuations; it is usually referred to as the "wave-noise". There is an important difference between Eqs. (1) \& (7), however, that deserves note. Einstein's expression is for the energy fluctuations of a particular electric field, that of a blackbody; Eq. (7) represents the measured photoelectron fluctuations obtained from detecting an arbitrary classical wave.

An important special case occurs when we consider a wave that has a constant amplitude with no fluctuations. In this case the distribution of integrated intensities is a delta function $p_{W}(W)=\delta(W-\bar{W})$, with $\langle W\rangle=\bar{W}$. Substituting this distribution into Eq. (5), we obtain for the distribution of 
photocounts

$$
P(n)=\frac{(\gamma \bar{W})^{n}}{n !} e^{-\gamma \bar{W}}
$$

This is a Poissonian distribution of counts, which is not surprising, as the assumptions that went into our classical model are exactly the assumptions necessary to ensure that electron emission is a Poissonian random process. [9]

Since the intensity of the wave is constant, the wave-noise is zero, and the mean and variance of the photocount distributions can be obtained from Eqs. (6) and (7) as

$$
\begin{aligned}
\langle n\rangle & =\gamma \bar{W} \\
\left\langle(\Delta n)^{2}\right\rangle & =\langle n\rangle .
\end{aligned}
$$

As can be seen in Eq. (9), the variance of the number of counts is equal to the mean, as expected for a Poissonian distribution. The noise level obtained in detecting this constant intensity signal, as expressed by the variance, is the minimum noise level allowed by classical theory for the detection of any optical signal of a given mean. This can be seen by examining Eq. (7); the wave-noise term is strictly positive, so the minimum noise will be obtained when the wave-noise is zero. This signal is said to be "shot-noise limited", and for a time it was believed that it was impossible to detect optical signals with less noise than this.

In semi-classical detection theory the optical signal is again treated as a classical wave, but now the emission of electrons in the detector is treated 
quantum mechanically. Since we are no longer treating the detector as a black box, we can derive the rate at which electrons are emitted from the detector, without having to resort to postulates. The first successful semiclassical detector model was put forth in 1964, and we will not go over this model in detail.[10, 11] It is enough to state that the semi-classical model shows explicitly that the probability that an electron will be emitted by the detector in a short time interval is proportional to the incident light intensity. In other words, the semi-classical model derives Eq. (2) from the quantum mechanical interaction of an atom and an electric field. The semiclassical model also shows that the detector efficiency is given by $\gamma=\eta_{d} /(h \nu)$, where $\eta_{d}$ is a dimensionless quantity known as the quantum efficiency of the detector. The rest of the assumptions in the classical model are the same as those of the classical model, and the results of the two models are identical. So, the semiclassical model also predicts the shot-noise as the minimum noise in the detection of a light signal.

\subsection{Quantum Theory}

In the quantum mechanical treatment, the electric field is no longer a classical random variable, but a quantum mechanical operator (for more details of field quantization, the reader is referred to Refs. $[11,12,13])$. The operator $\hat{E}$ for a plane-wave mode of an electric field is

$$
\hat{E}=i \omega\left(\frac{\hbar}{2 V \varepsilon_{0} \omega}\right)^{\frac{1}{2}}\left[\hat{a} e^{i(\vec{k} \cdot \vec{r}-\omega t)}-\hat{a}^{\dagger} e^{-i(\vec{k} \cdot \vec{r}-\omega t)}\right]
$$


Here, $\vec{k}$ is the wavevector of the field, $\omega$ is its angular frequency, and $V$ is the quantization volume. The operators $\hat{a}$ and $\hat{a}^{\dagger}$ are annihilation and creation operators for the photons in the field mode, in analogy to the raising and lowering operators of the quantum mechanical harmonic oscillator. The fact that these operators appear in the electric field is not surprising, since an electric field is an oscillating wave. Indeed, a single mode of an electric field is a quantum mechanical harmonic oscillator, and everything we know about simple harmonic oscillators can be used to describe the behavior of an electric field. The creation and annihilation operators satisfy the commutation relation

$$
\left[\hat{a}, \hat{a}^{\dagger}\right]=1
$$

and the operator which yields the number of photons in the field is $\hat{n}=\hat{a}^{\dagger} \hat{a}$.

Having defined the operators which describe an electric field, we must now describe the states that the field can occupy. A convenient set of orthonormal basis states are the number states, or Fock states, $|n\rangle$ which are eigenstates of the number operator

$$
\hat{n}|n\rangle=n|n\rangle
$$

with eigenvalues $n$ being positive integers or zero. These states yield the familiar Hermite-Gaussian wavefunctions of the harmonic oscillator. The action of the creation and annihilation operators on these states is given by

$$
\begin{aligned}
\hat{a}^{\dagger}|n\rangle & =\sqrt{n+1}|n+1\rangle, \\
\hat{a}|n\rangle & =\sqrt{n}|n-1\rangle .
\end{aligned}
$$


Given a number state $|n\rangle$, the expectation value for the electric field associated with this state can be calculated as

$$
\langle\hat{E}\rangle=\langle n|\hat{E}| n\rangle=0 .
$$

The fact that the mean field for light in a number state is zero for all times indicates that this light differs significantly from light which can be described by a classical theory in which the field is sinusoidally oscillating. However, the fact that the mean field is zero does not mean that there is no energy (photons) associated with light in a number state. If an electric field is in a state $|\psi\rangle$, then the probability that the field will contain $n$ photons is given by

$$
P(n)=|\langle n \mid \psi\rangle|^{2} .
$$

Thus, if the field is in the number state $|m\rangle$, the photon number distribution is

$$
P(n)=|\langle n \mid m\rangle|^{2}=\delta_{n m},
$$

where $\delta_{n m}$ is the Kronecker delta function. In this case the field contains a definite number of photons.

Again, we are most interested in moments of the photon number distribution and not the distribution itself. For a field in the state $|\psi\rangle$, the mean and the variance of the photon number are

$$
\begin{aligned}
\langle\hat{n}\rangle & =\langle\psi|\hat{n}| \psi\rangle \\
\left\langle(\Delta \hat{n})^{2}\right\rangle & =\left\langle(\hat{n}-\langle\hat{n}\rangle)^{2}\right\rangle=\left\langle\psi\left|\hat{n}^{2}\right| \psi\right\rangle-\langle\psi|\hat{n}| \psi\rangle^{2} .
\end{aligned}
$$


For the number state $|\psi\rangle=|m\rangle$, the mean and variance are

$$
\begin{aligned}
\langle\hat{n}\rangle & =m \\
\left\langle(\Delta \hat{n})^{2}\right\rangle & =0,
\end{aligned}
$$

consistent with a field containing a definite number of photons and having no fluctuations. If we had a perfect detector, for which every incident photon was converted to a photoelectron, this field would show zero noise in its photocount distribution, in stark contrast to the classical and semi-classical results. Below we will explore how close to this perfect result we can come.

There is one other set of field states of interest to us here, and these are the coherent states $|\alpha\rangle \cdot[14]$ Coherent states are eigenstates of the annihilation operator

$$
\hat{a}|\alpha\rangle=\alpha|\alpha\rangle .
$$

Because the operator $\hat{a}$ is not Hermitian, the eigenvalues $\alpha$ are complex. By solving the eigenvalue problem of Eq. (22), one can show that the eigenvalues (referred to as the amplitude of the coherent state) can be any complex number. The eigenstates can be expressed in terms of the number state basis as

$$
|\alpha\rangle=e^{-|\alpha|^{2} / 2} \sum_{n} \frac{\alpha^{n}}{\sqrt{n !}}|n\rangle .
$$

The coherent states are of interest because they are the quantum mechanical state which is the closest equivalent to a classical electromagnetic wave. 
If the field is in a coherent state, the expectation value of the electric field operator is

$$
\langle\hat{E}\rangle=E_{0} \cos (k \cdot r-\omega t)
$$

indicating that the mean field has a particular amplitude and phase, like that of a constant amplitude classical wave. By using Eqs. (16) \& (23), it is seen that for a field in the state $|\alpha\rangle$ the photon number distribution is

$$
P(n)=\frac{|\alpha|^{2 n}}{n !} e^{-|\alpha|^{2}}
$$

Thus, coherent states contain a Poissonian distribution of photons, with a mean and variance of

$$
\begin{aligned}
\langle\hat{n}\rangle & =|\alpha|^{2} \\
\left\langle(\Delta \hat{n})^{2}\right\rangle & =|\alpha|^{2}=\langle\hat{n}\rangle .
\end{aligned}
$$

If we had a perfect detector, the distribution of photocounts from a coherent state beam of light would be Poissonian, in agreement with classical and semiclassical detection theories. This indicates that the results of the classical and semi-classical theories for the case of a constant amplitude wave can be reproduced in the quantum theory by using a coherent state for the state of the light field.

Now we must address the problem of detecting the above described quantum mechanical light fields. The full quantum theory of photodetection was developed by Kelley and Kleiner in 1964, and here we present only as much 
of the theory as we need to understand our experiment.[15, 11, 12] The essential point of quantum detection theory is that a quantum detector converts incident photons to photoelectrons. As alluded to above, a perfect detector does this with $100 \%$ efficiency, and hence the photoelectron statistics exactly mimic the photon statistics. Inefficiencies in the detector degrade this 1:1 correspondence between photons and photoelectrons, and tend to mask quantum mechanical effects. In order to understand the results of our experiment, it is essential to understand how the detector efficiency affects the photocount statistics. Rather than directly treating inefficiencies in the detector, which is mathematically involved, we will use a simpler, equivalent model. We will model the detection process as involving a fictitious beamsplitter which causes the incident light beam to suffer a certain amount of loss, followed by a perfect detector which measures the statistics of this loss-modified beam.

In Fig. 1 we show a diagram of a beamsplitter, and we have labeled the incoming and outgoing fields by their corresponding annihilation operators. The light field we are attempting to measure is in mode $\hat{a}_{1}$. The beamsplitter introduces a loss (the light which escapes through $\hat{a}_{4}$ ), which serves to model the imperfect quantum efficiency of the detector, and the attenuated mode $\hat{a}_{3}$ is detected by a perfect detector. Thus, by determining the photon statistics of $\hat{a}_{3}$, we determine the photocount statistics recorded by an imperfect detector.

The output field operators must be related to the input operators by the 
reflection and transmission coefficients of the electric field $r_{1}, r_{2}, t_{1}, t_{2}$ :

$$
\begin{aligned}
& \hat{a}_{3}=t_{1} \hat{a}_{1}+r_{2} \hat{a}_{2} \\
& \hat{a}_{4}=r_{1} \hat{a}_{1}+t_{2} \hat{a}_{2} .
\end{aligned}
$$

In general the coefficients $r_{1}, r_{2}, t_{1}, t_{2}$ are complex numbers, and these coefficients must satisfy certain constraints.[11] For example, energy must be conserved, so the total output energy must equal the total input energy (assuming a nonabsorbing beamsplitter). Also, each of the four fields are in modes which can be independently measured; it is therefore necessary that the operators for each of these modes commute. In particular, it must be true that

$$
\left[\hat{a}_{i}, \hat{a}_{j}^{\dagger}\right]=\delta_{i j}
$$

A solution that satisfies all of the necessary constraints is one where $r$ and $t$ satisfy $t=t_{1}=t_{2}, r=-r_{1}=r_{2}$ and $|t|^{2}+|r|^{2}=1$. This makes the relations in Eq. (28)

$$
\begin{aligned}
& \hat{a}_{3}=t \hat{a}_{1}+r \hat{a}_{2} \\
& \hat{a}_{4}=t \hat{a}_{2}-r \hat{a}_{1} .
\end{aligned}
$$

By examining Eq. (30), we see that the statistics of $\hat{a}_{3}$ will depend not only on the field of primary interest $\hat{a}_{1}$, but also on $\hat{a}_{2}$. In a classical treatment we can completely ignore the mode $\hat{a}_{2}$, since the only light incident on the detector comes from $\hat{a}_{1}$. Quantum mechanically $\hat{a}_{2}$ cannot be ignored, as it is necessary 
to ensure that the commutation relations [Eq. (29)] are satisfied. The question arises then, what do we specify for the state of the field in mode $\hat{a}_{2}$ ? Since the only energy incident on the detector comes from $\hat{a}_{1}, \hat{a}_{2}$ must be in a zero energy state. Thus, $\hat{a}_{2}$ is in a so-called vacuum state $|0\rangle$, which is either number state with $n=0$, or a coherent state with $\alpha=0$. Since the statistics of $\hat{a}_{3}$ depend on the states of two modes, we must label these states by two quantum numbers (i.e. : the state $\left|n_{1} ; 0\right\rangle$ indicates that $\hat{a}_{1}$ is in a number state containing $n_{1}$ photons, while $\hat{a}_{2}$ is in a vacuum state.)

We are now ready to calculate the photon statistics of mode $\hat{a}_{3}$. The operator for the number of photons in mode $\hat{a}_{3}$ is

$$
\begin{aligned}
\hat{n}_{3}=\hat{a}_{3}^{\dagger} \hat{a}_{3} & =\left(t^{*} \hat{a}_{1}^{\dagger}+r^{*} \hat{a}_{2}^{\dagger}\right)\left(t \hat{a}_{1}+r \hat{a}_{2}\right) \\
& =|t|^{2} \hat{n}_{1}+|r|^{2} \hat{n}_{2}+t^{*} r \hat{a}_{1}^{\dagger} \hat{a}_{2}+r^{*} t \hat{a}_{2}^{\dagger} \hat{a}_{1} .
\end{aligned}
$$

If $\hat{a}_{1}$ is in a number state, the mean number of detected photons is then

$$
\begin{aligned}
\left\langle\hat{n}_{3}\right\rangle & =\left\langle n_{1} ; 0\left|\left[|t|^{2} \hat{n}_{1}+|r|^{2} \hat{n}_{2}+t^{*} r \hat{a}_{1}^{\dagger} \hat{a}_{2}+r^{*} t \hat{a}_{2}^{\dagger} \hat{a}_{1}\right]\right| n_{1} ; 0\right\rangle \\
& =|t|^{2} n_{1} \\
& =\eta_{d} n_{1} .
\end{aligned}
$$

The quantum efficiency of the detector $\eta_{d}$ is the fraction of input photons converted to photoelectrons, and is given by the intensity transmission of the beamsplitter $\eta_{d}=|t|^{2}$. A little algebra shows that the variance of the photocounts when mode $\hat{a}_{1}$ is in a number state is

$$
\left\langle\left(\Delta \hat{n}_{3}\right)^{2}\right\rangle=|r|^{2}|t|^{2} n_{1}=\left(1-|t|^{2}\right)|t|^{2} n_{1}
$$




$$
=\left(1-\eta_{d}\right) \eta_{d} n_{1}=\left(1-\eta_{d}\right)\left\langle\hat{n}_{3}\right\rangle .
$$

In the limit of perfect detection $\left(\eta_{d} \rightarrow 1\right)\left\langle\left(\Delta \hat{n}_{3}\right)^{2}\right\rangle \rightarrow 0$, as would be expected for a noiseless input state. We see from Eq. (33), however, that for less than unity detection efficiency there will be some fluctuation in the photocounts, despite the fact that the input light field is noiseless. One way to interpret this is that the transmission of a photon through the beamsplitter is a random process. Each photon incident on the beamsplitter will be transmitted with probability $|t|^{2}$, while it will be reflected with probability $1-|t|^{2}$. On average, $|t|^{2} \times 100 \%$ of the photons will be detected [c.f. Eq. (32)], but the number of detected photons will fluctuate from measurement to measurement.

For a coherent state input, the mean and variance of the detected signal are found to be

$$
\begin{aligned}
\left\langle\hat{n}_{3}\right\rangle & =\left\langle\alpha_{1} ; 0\left|\left[|t|^{2} \hat{n}_{1}+|r|^{2} \hat{n}_{2}+t^{*} r \hat{a}_{1}^{\dagger} \hat{a}_{2}+r^{*} t \hat{a}_{2}^{\dagger} \hat{a}_{1}\right]\right| \alpha_{1} ; 0\right\rangle \\
& =|t|^{2}\left|\alpha_{1}\right|^{2} \\
& =\eta_{d}\left|\alpha_{1}\right|^{2},
\end{aligned}
$$

and

$$
\begin{aligned}
\left\langle\left(\Delta \hat{n}_{3}\right)^{2}\right\rangle & =|t|^{2}\left|\alpha_{1}\right|^{2}=\eta_{d}\left|\alpha_{1}\right|^{2} \\
& =\left\langle\hat{n}_{3}\right\rangle .
\end{aligned}
$$

Thus, an attenuated coherent state has a photon number variance equal to its mean, just as it had before attenuation. 
A useful measure of the noise strength is the Fano factor, which is the variance of the detected signal normalized by the shot-noise $\left\langle(\Delta \hat{n})_{s n}^{2}\right\rangle$ of a signal with the same mean signal strength. The shot-noise variance [c.f. Eq. (9)] is equal to the mean signal. We denote the Fano factor for an incident number state incident as $F_{n s}$, and it is given by

$$
\begin{aligned}
F_{n s} & =\frac{\left\langle\left(\Delta \hat{n}_{3}\right)^{2}\right\rangle}{\left\langle\left(\Delta \hat{n}_{3}\right)_{s n}^{2}\right\rangle}=\frac{\left\langle\left(\Delta \hat{n}_{3}\right)^{2}\right\rangle}{\left\langle\hat{n}_{3}\right\rangle} \\
& =\left(1-\eta_{d}\right) .
\end{aligned}
$$

As can be seen in Eq. (36), $F_{n s}$ depends only on the quantum efficiency of the detector.

For a coherent state input the Fano factor is

$$
F_{c s}=\frac{\left\langle\left(\Delta \hat{n}_{3}\right)^{2}\right\rangle}{\left\langle\hat{n}_{3}\right\rangle}=1,
$$

which is a constant, independent of the efficiency. Since the quantum mechanical results for a coherent state are the same as those of classical and semi-classical theories for a constant amplitude wave, these theories would predict a Fano factor of unity for detecting a classical wave with no amplitude fluctuations.

In brief, classical and semi-classical detection theories predict that the photocount statistics for a constant classical wave will be Poissonian. This leads to a minimum noise level, the shot-noise level, for a field of a given amplitude. The detected Fano factor of this wave will be equal to 1, independent of the 
efficiency of the detector. In the quantum theory, the coherent state is a light field which when detected has a Fano factor of 1 . However, quantum theory shows that for certain light fields it is possible to perform optical detection with noise lower than the shot-noise level; the amount of noise reduction depends on the efficiency of the detection process. When a detected field has a Fano factor of less than 1, as is the case of a light field in an attenuated number state, then the field is said to be sub-Poissonian. Such a field is only describable using quantum mechanics and has no classical analog.

\section{Experiment}

In our experiment the noise is measured by observing the electrical current generated by a photodetector. Measurements are made at frequency $f$ within a bandwidth $\Delta f$, using either a spectrum analyzer or a digitizing oscilloscope. Here we relate the measurements we perform to the theory presented above.

The mean current $\langle i\rangle$ from the photodetector is simply the flux of electrons in a time interval $T$. Assuming most of the electrons in our current are photoelectrons (i.e.: any "dark" current is much smaller than the photocurrent), the mean current can be expressed in terms of the mean number $\langle n\rangle$ of photoelectrons being counted as

$$
\langle i\rangle=\frac{\langle n\rangle e}{T}
$$

where $e$ is the charge of an electron. The variance of the current will be 


$$
\begin{aligned}
\left\langle(\Delta i)^{2}\right\rangle & =\left\langle i^{2}\right\rangle-\langle i\rangle^{2} \\
& =\left(\frac{e}{T}\right)^{2}\left[\left\langle n^{2}\right\rangle-\langle n\rangle^{2}\right] \\
& =\left(\frac{e}{T}\right)^{2}\left\langle(\Delta n)^{2}\right\rangle \\
& =(2 e \Delta f)^{2}\left\langle(\Delta n)^{2}\right\rangle,
\end{aligned}
$$

where $\Delta f=\frac{1}{2 T}$ is the electrical bandwidth of the detection system. The noise is broadband, with equal amplitude for all frequencies (within the limits of the electrical response of the amplification system and the other electronic components). Thus, the variance of the current fluctuations at at a particular frequency is simply proportional to the variance of the photoelectron counts.

The variance for the current as shown in Eq. (39) is dependent upon the variance in the number of photoelectrons, which is in turn dependent upon the state of the light. Using the results from Eqs. (33) and (35) for the variance in $n$, we can say

$$
\left\langle(\Delta i)^{2}\right\rangle= \begin{cases}(2 e \Delta f)^{2}\langle n\rangle & \text { for coherent state signal } \\ (2 e \Delta f)^{2}\langle n\rangle(1-\eta) & \text { for number state signal. }\end{cases}
$$

In Eq (40) we have substituted $\eta$, the total system efficiency, for $\eta_{d}$, the detector efficiency. The total efficiency is the product of the efficiencies of all the processes between the generation of the light, and its conversion to photoelectrons (i.e. the efficiency of the LED, the collection efficiency of the optics, and the efficiency of the photodetector). In making this substitution, we are 
assuming that all of these losses can be modeled by a beamsplitter loss as described above. The beamsplitter is a good model for losses due to collection and detector inefficiencies. However, the beamsplitter model is not necessarily very good in describing the efficiency of converting electrons to photons in the LED. This is because the electrons in the LED are fermions, and they do not partition the same way at a junction as the bosons in our beamsplitter model. [6] Despite this limitation, we can measure the drive current to the LED and the average photocurrent, and then calculate what we call the current-to-current conversion efficiency. This is a reasonable estimate of the overall system efficiency $\eta$.

Shown in Fig. (2) is a schematic overview of the experimental apparatus. The two current sources are outlined with dashed lines and are marked as the constant current source (CCS) and the Poissonian current source (PCS); the LED can be driven with either one of these two current sources. The LED (Hammamatsu L2656) is a high efficiency GaAlAs semi-conductor emitter with a specified quantum efficiency of 0.22 photons-per-electron at a center wavelength of $890 \mathrm{~nm}$. The LED is mounted on an aluminum block, which is in turn mounted to a thermo-electric cooler (TEC). The temperature of the LED is controlled by a laser-diode temperature controller (ILX LDT-5525). The photodiode ( Hammamatsu S5107, labeled PD B in Fig. 2) has a large active area of $1 \mathrm{~cm}^{2}$ and a specified quantum efficiency of 0.93 . In order to collect as much light as possible, the LED is positioned directly in front of 
the photodiode. The current from the photodiode is converted to a voltage with a transimpedence amplifier, and then further amplified. [16] The noise power is measured with a Hewlett Packard HP-8590L Spectrum analyzer. The spectrum anlayzer gives the electrical power of the signal as a function of frequency.

The essence of this experiment is that we wish to detect a signal below the shot-noise level. In order to do this, we must be able to determine exactly what the shot-noise level is. In the theoretical discussion above, we showed that the shot-noise level was given by the mean number of detected photoelectons. It is also equal to the variance of a coherent state signal field (i.e., a field with Poissonian statistics). Thus, if we can generate a field with Poissonian statistics and measure its noise level, we can compare other measured fields to it. In order to generate a field with Poissonian statistics, we drive the LED with a Poissonian current source. [6]

In our experimental setup, the PCS consists of a halogen lamp which illuminates two reverse biased photodiodes (UDT 10-DP, labeled PD A in Fig. 2). The current produced by these photodiodes is used to drive the LED. The variance in this current, $\left\langle\left(\Delta i_{P C S}\right)^{2}\right\rangle$, can be expressed as either, $\left\langle\left(\Delta i_{P C S}\right)^{2}\right\rangle=\left\langle i_{P C S}\right\rangle$ or $\left\langle\left(\Delta i_{P C S}\right)^{2}\right\rangle=(1-\eta)\left\langle i_{P C S}\right\rangle$, depending upon the state of the light incident on the diodes. However, as the efficiency $\eta$ approaches zero (while holding the mean current fixed), the variance in the photocurrent will be approximately the same regardless of the state of the incident light. If the 
efficiency approaches zero, the current noise will approach the shot-noise as defined in Eq (9). In the PCS, the halogen lamp was driven with approximately 3 amps of current, which then produced approximately $30 \mathrm{~mA}$ of current in the photodiodes. Using the current-to-current conversion ratio, $\eta=0.01$, the noise in the current generated by the photodiodes is a good approximation of the shot noise.

There are two different purposes for photodiodes in the experimental setup, and some confusion could arise concerning to which photodiode we are refering. The photodiodes in the PCS (labeled PD A)are used to generate a current with Poissonian statistics, which is then used to drive the LED. Hence, any references to the current from these photodiodes will hereafter be specified as the driving current. The photodiode (labeled PD B) in the detection system is used to measure the noise of the light incident upon it. The current from this photodiode will hereafter be specified simply as the photocurrent.

We must be certain that the light statistics we use as our reference are Poissonian, and not super-Poissonian. If the light statistics are super-Poissonian, the amount of noise reduction observed (relative to the shot-noise reference level) would be apparently greater than the actual noise reduction. To verify that the PCS is actually creating light with shot-noise variance, there are two things which can be experimentally checked.

(1) Equation (40) gives the variance in the photocurrent as a linear function of the mean photocurrent. In our experiments, the variance is measured 
as electrical noise power. From Eq. (7) we see that any additional fluctuations present on the light beam will be quadratic in the optical power (or photocurrent). Thus, if the photocurrent noise is linear with the mean photocurrent, we can be confident that the noise power is due to the shot-noise, and not other noise sources within the electronics of the detection and amplification system. Also, by ensuring the linearity of the noise for large mean photocurrents, we can be sure the detection system is not being saturated at higher DC levels.

This test was performed by shining a red filtered white light source on the detector, and then changing the amount of light falling on the detector to vary the DC photocurrent. The plot of the noise power as a function of the DC photocurrent is shown in figure (3). The linearity of this plot indicates that the noise output from the detection and amplification system is shot-noise and not spurious noise. The upper range of the plot also indicates that the detector is not being saturated at DC currents of almost $10 \mathrm{~mA}$, which is a higher photocurrent than used in the experiment. This linearity test was then repeated using the LED driven by the PCS as the light source; this produced the same results as the white light source.

(2) Equation (37) gives the normalized noise (Fano factor) for light with Poisson statistics as being constant with efficiency. In contrast, Eq. (36) states that the noise for a number state field is a linear function of 
the efficiency, with the noise decreasing as the efficiency increases. The measured normalized variance in the photocurrent with the LED driven by the PCS should thus be constant with varying efficiency.

This test was performed by measuring the noise at a fixed photocurrent, as the efficiency was varied. The efficiency was decreased between measurements by simply increasing the separation distance from the LED to the photodiode; the driving current was then adjusted to give the same mean photocurrent for all of the measurements. The efficiency was measured as the ratio of the photocurrent to the driving current. As can be seen in Fig. (4), the noise is constant with varying efficiency (to within our measurement precision), indicating that the light statistics are indeed Poissonian when the LED is driven by the PCS.

The constant current source consists of a voltage source ( a rechargeable lead-acid battery ) in series with a high impedence (a resistance of $274 \Omega$ ). For additional noise suppresion, an inductor, a surge suppresor, and an EMI filter were placed between the battery and the resistor. The CCS was used to generate current with variance well below the shot-noise. The current in the CCS is not subject to the shot-noise limitations, because the electrons which make up the current are fermions (i.e. they are not independent particles). [17] Since the drive current is essentially noise free, the light output will be noise free within the limits imposed by the efficiency of the LED. So the light from the LED driven by the CCS should upon detection show variance below 
the shot noise limit as determined by $\operatorname{Eq}(36) .[4,5,6]$

We have shown that the PCS produces light with shot noise variance. Thus the Fano factor for a measured signal can be obtained by normalizing the noise in the measured signal to the noise produced by the light from the PCS (assuming the same mean photocurrent for the measured signal and the PCS signal).

Shown in Fig. (5) are the spectrum analyzer traces for the photocurrent noise for light produced by the PCS (a), the CCS (b), and the electronic noise with no light incident on the photodiode (c). The noise for the PCS and CCS were measured at the same photodiode mean current. The average noise reduction seen from the $\mathrm{PCS}$ to the $\mathrm{CCS}$ is $0.7 \mathrm{~dB}$ (i.e., light with a Fano factor of 0.85 ). Based on an overall efficiency of 0.18 , one would predict a Fano factor of 0.82 .

Shown in Fig. (6) are the measured Fano factor (circles) and the theoretical Fano factor (solid line, determined from measured efficiency) as a function of the efficiency. We see that the Fano factor decreases with increasing efficiency, exactly as we would expect from the theoretical result in Eq. (36). We see that the measurements give Fano factors consistently higher than would be predicted by the measured overall efficiency. This same discrepancy has been seen by other authors. [6]

In a separate experiment, the LED was cooled to $100 \mathrm{~K}$ using a cryostat in order to increase the efficiency of the LED. The efficiency of the LED at 
$100 \mathrm{~K}$ was approximately twice its value at $283 \mathrm{~K}\left(10^{\circ} \mathrm{C}\right)$. However, the light collection efficiency was lower with the LED inside the cryostat, because the photodetector was outside the cryostat and we were unable to image all of the light emitted from the LED onto the face of the detector. With the LED (at $100 \mathrm{~K}$ ) in the cryostat, we were able to increase the overall efficiency to $20 \%$, an improvement over the best efficiency we could obtain with the LED (at $283 \mathrm{~K}$ ) positioned directly in front of the photodetector $(18 \%)$. We were thus able to observe a noise reduction of $1.0 \mathrm{~dB}$ with the LED at $100 \mathrm{~K}$. It may be interesting to note that with the LED at $100 \mathrm{~K}$ the amount of noise reduction we observed agreed quite well with the theoretical value of $1.0 \mathrm{~dB}$ predicted from the measured current-to-current conversion efficiency, while the agreement at near room temperature is not as good.

In a last experiment, we wanted to see if it would be possible to reduce the cost of the experiment even further, and use only equipment already found in most undergraduate laboratories. The two pieces of equipment that we have used above which are somewhat specialized and expensive are the temperature controller and the RF spectrum analyzer. In this experiment we have run the LED at room temperature without temperature control. Since we were not cooling the LED very much anyway, this has no noticeable effect on the results. Also, we have replaced the spectrum analyzer in our apparatus with a digital oscilloscope (Tektronix 2232), in order to use a more widely available data acquisition instrument. 
We acquire 1024 points per trace with the digital scope, at a sample rate of $5 \mathrm{MHz}$. For each trace we transfer the data to the computer, then compute the power spectrum of the signal using a fast Fourier transform (FFT). With our sampling parameters the power spectrum has a resolution of approximately $5 \mathrm{kHz}$, and a maximum frequency of $2.5 \mathrm{MHz}$. To decrease the sampling noise, we average the power spectra from 400 traces; this is equivalent to video averaging on the spectrum analyzer. Our sample rate is above the high frequency cutoff of the detector, which is necessary to ensure that the signal does not experience aliasing. If the sample rate is set to low, high frequency noise is aliased into lower frequencies, and the noise spectrum is flat all the way down to DC. With proper sampling the noise decreases at low frequencies because of the high-pass filter in the detector circuit.

Shown in Fig. (7) are noise spectra taken with the Poissonian current source and the constant current source. The electronic noise is approximately $10 \mathrm{~dB}$ below both of these curves. Between $200 \& 500 \mathrm{kHz}$, the average noise reduction is found to be $0.6 \mathrm{~dB}$ (Fano factor of 0.87 ), while the measured current-to-current conversion efficiency would predict a noise reduction of 0.8 $\mathrm{dB}$ (Fano factor of 0.83 ). Thus, it is possible to use a digital scope to observe this quantum effect, obviating the need for a relatively specialized RF spectrum analyzer if one is not available. Further averaging will reduce the noise in the power spectra shown in Fig. (7), but acquiring, transferring and processing the 400 traces for each of the curves shown requires approximately 20 minutes. 
Taking data with the spectrum analyzer is much quicker, since one can narrow in on the frequency range of interest, and hence acquire data quickly.

\section{Conclusions}

The theory and experiment presented above explore the statistical properties of photocurrents. Shot-noise is found to be a limitation for the noise in a

photocurrent, while quantum theory predicts no such limitation. The experiment measured a photocurrent with a noise power below the shot-noise level, in agreement with the quantum theory but not describable using classical or semi-classical detection theory. This experiment thus provides an accessible way for undergraduates to experimentally observe a purely quantum mechanical effect.

\section{Acknowledgements}

We thank Jean-François Roch of the Institut d'Optique Théorique for the detection circuit, and Dan Kilper of the University of Michigan for the LED current source circuit. This work was supported by Reed College. 


\section{References}

[1] A. Einstein, "Zum Gegenwärtigen Stand des Strahlungsproblems (On the present status of the radiation problem)", Phys. Zeit. 10, 185-193 (1909).

[2] A. Einstein, " Über die Entwickelung unserer Anschauungen über das Wesen und die Konstitution der Strahlung (On the development of our views concerning the nature and constitution of radiation)", Phys. Zeit. 10, 817-826 (1909).

[3] We will consistently adopt the notation that $\langle A\rangle$ denotes the mean of a classical random variable $A$, while $\left\langle(\Delta A)^{2}\right\rangle$ denotes its variance.

[4] P.R. Tapster, J.G. Rarity and J.S. Satchell, "Generation of sub-Poissonian light by high-efficiency light-emitting diodes", Europhys. Lett. 4, 293-299 (1987).

[5] P.J. Edwards, "Sub-Poisson light from GaAlAs infrared emitting diodes", Int. J. Optoelectronics 6, 23-28 (1991).

[6] H.-A. Bachor, P. Rottengatter and C.M. Savage, "Correlation effects in light sources with high quantum efficiency", App. Phys. B 55, 258-264 (1992).

[7] P. Koczyk, P. Wiewiór and C. Radzewicz, "Photon counting statisticsundergraduate experiment", Am. J. Phys. 64, 240-245 (1996). 
[8] L. Mandel, "Fluctuations of photon beams: the distribution of the photoelectrons", Proc. Phys. Soc. (London) 74, 233-243 (1959).

[9] Joseph W. Goodman, Statistical Optics (John Wiley and Sons, New York, 1985).

[10] L. Mandel, E.C.G. Sudarshan and E. Wolf, "Theory of photoelectric detection of light fluctuations", Proc. Phys. Soc. London 84, 435-444 (1964).

[11] L. Mandel and E. Wolf, Optical Coherence and Quantum Optics (Cambridge Univ. Press, Cambridge, 1995).

[12] Rodney Loudon, The Quantum Theory of Light, 2nd ed. (Clarendon, Oxford, 1983).

[13] I.D. Johnston, "Photon states made easy: A computational approach to quantum radiation theory", Am. J. Phys. 64, 245-255 (1996).

[14] For a more complete discussion of the properties of the coherent states, see $[11,13]$.

[15] P.L. Kelley and W.H. Kleiner, "Theory of electromagnetic field measurement and photoelectron counting", Phys. Rev. 136, 316-334 (1964).

[16] For a copy of the circuit diagram, please send a self-addressed, stamped envelope to : Mark Beck, Dept. of Physics, Whitman College, Walla Walla, WA 99632. 
[17] P. Horowitz and W. Hill, The Art of Electronics, 2nd ed. (Cambridge University Press, Cambridge, 1989), pp 431-432. 


\section{Figure Captions}

Figure 1: Beamsplitter model of photodetector, the operators for the individual fields are marked as $\hat{a}_{i}$.

Figure 2: Overview of the experimental apparatus: The current sources are outlined in dashed lines. PCS is the Poissonian current source, while CCS is the Constant current source; we can alternate between the current sources. In the PCS, light from the lamp is detected by the photodiodes labeled PD A. The light from the LED is detected with the photodiode labeled PD B. We

measure the average current through PD B by measuring the voltage at the point labeled DC. The noise power is measured after amplification at the point labeled RF.

Figure 3: Experimental (points) and linear fit (solid line) of noise power as a function of mean photocurrent for red filtered white light incident on the photodiode. 
Figure 4: Photocurrent noise power for light from the LED driven by the PCS as a function of overall system efficiency. The points are measured values, while the horizontal line represents the average of the measured points.

Figure 5: Measured photocurrent noise power for the LED driven by the PCS (a), $\operatorname{CCS}(\mathrm{b})$, and electronic noise(c). The mean photocurrent was the same (6.85 $\mathrm{mA})$ when the LED was driven by both the PCS and the CCS. The electronic noise was measured with no light incident on the photodiode. The average noise reduction between the PCS and the CCS is $0.7 \mathrm{~dB}$. For each of these curves, the resolution bandwidth was $1.0 \mathrm{kHz}$, the video bandwidth was $30 \mathrm{~Hz}$, and each trace was averaged 50 times.

Figure 6: Experimental (points) and theoretical (solid line) Fano factor as a function of current-to-current conversion efficiency, $\eta$.

Figure 7: Photocurrent noise power for the LED driven by the PCS [solid line](a) and CCS [dashed line](b) as measured using the digitizing oscilloscope. Average noise reduction between 200 and $500 \mathrm{kHz}$ was $0.6 \mathrm{~dB}$. Each curve is an average of 400 traces, and the resolution is $5 \mathrm{kHz}$. 